\title{
A model study on superfluidity of a unitary Fermi gas of atoms interacting with a finite-ranged potential
}

\author{
Subhanka Mal and Bimalendu Deb \\ School of Physical Sciences, Indian Association for the Cultivation of Science, Jadavpur, Kolkata 700032, India.
}

\begin{abstract}
We calculate Bardeen-Cooper-Schrieffer (BCS) state of a unitary Fermi gas of atoms interacting with the finite-ranged Jost-Kohn potential which has been recently shown to account for the resonant interactions [2019 J. Phys. B: At. Mol. Opt. Phys. 52, 165004]. Using exact scattering solution of the potential, we derive two-body T-matrix element which is employed to construct the BCS Hamiltonian in momentum space. We present results on the energy- and range-dependence of the pairing gap and superfluid density and the range-dependence of the chemical potential for a wide variation of the scattering length including the unitary regime. In the zero range limit our calculated gap at the Fermi energy is found to be nearly equal to that calculated in mean-field theory with contact potential. The mean gap averaged over the full width at half maximum of the gap function in the zero range and unitary limits is found to be $0.42 E_{F}$ which is quite close to the recent result of the quantum Monte Carlo simulation [2018 Phys. Rev.A 97, 013601]. The chemical potential in the zero range limit also agrees well with that for the contact potential.
\end{abstract}

PACS numbers:

\section{INTRODUCTION}

The experimental advancement in many-body quantum physics [1] with trapped ultracold atoms over the last two decades has opened new perspectives in both bosonic and fermionic superfluidity. In particular, the studies of superfluidity with a Fermi gas of atoms have implications in understanding the superconductivity of condensed matter systems, superfluidity of He-3 liquid and superconductivity or superfluidity of nuclear matter of astrophysical origin. It is expected that quantum simulations of various types of fermionic superfluidity using trapped atomic gases with control over atom-atom interactions, trap dimensionality and atomic density will provide new insight into electronic superconductivity and nuclear superfluidity. Superfludity and superconductivity occur due to the spontaneous breaking of continuous symmetry resulting in the appearance of long wave-length Goldstone modes which are also known as Bogoliubov-Anderson modes. While Goldstone modes in charged superconductors become ill-defined due to Coloumb interactions, such modes in neutral superfluidity are expected to be well-defined and detectable. Bogoliubov-Anderson modes have been recently measured in a superfluid Fermi gas of atoms [2, 3]. Thus the charge neutrality of atomic superfluidity offers some advantages in exploring fundamental aspects of quantum many-body physics. On the other hand, the aspects of superconductivity that are intrinsically connected with charge or gauge fields are not available with the neutral systems. Nevertheless, in recent times, artificial gauge fields for neutral atomic gases are created by optical manipulations of atomic internal as well as center-of-mass degrees-offreedom [4].

Bardeen-Cooper-Schrieffer (BCS) [5] type or standard superconductivity in electrons results from the phononmediated electron-electron attraction near the Fermi surface. This attractive interaction overcomes the Coulomb repulsion between the electrons at low energy, resulting in the formation of Cooper pairs which then constitute the many-body BCS state. Fermi superfluidity in trapped atomic gases arises when the atom-atom interaction is tuned towards attractive side by a magnetic Feshbach resonance [ 6 ]. In general, the ultracold atomic gases that are currently being experimentally studied are sufficiently dilute such that the range of interaction between the atoms is very small compared to the interatomic separation. Therefore, the range is generally neglected and the interaction is approximated as a contact potential expressed in terms of the $s$-wave scattering length $a_{s}$. A magnetic field is used to tune $a_{s}$ across the Feshbach resonance. A degenerate Fermi gas is transformed into BCS state by tuning $a_{s}$ towards small negative value for which the effective atom-atom interaction becomes attractive. Near the resonance where $a_{s}$ is large negative, the gas becomes strongly interacting leading to resonance superfluidity [7] that is characterized by the unitarity of the scattering $S$-matrix. On the positive side of $a_{s}$ across the resonance, the Cooper pairs may be transformed into bosonic diatomic molecules which can condense into a Bose-Einstein condensate. Such unitary regime and crossover physics may be available with color superconductivity in quantum chromodynamics and neutral superfludity of neutron stars.

Given the current state of the art in the experimental cold atom physics, an experimental attempt towards simulating neutron superfludity appears to be a difficult task. However, the recent successful observations of BCS-BEC crossover open prospect for simulating nuclear physics $[8-12]$. The effective range of interaction plays an important role in manybody quantum dynamics when it becomes comparable to inter-particle separation [13 [16]. In recent times, there has 
been considerable research interests to simulate dilute nuclear matter [17] such as proto-neutron stars [18, 19]. From a theoretical point of view, most of the works have been carried out by numerical experiments i.e., by Monte-Carlo simulations [20] or using density functional theory [21]. The effects of the finite range of interaction on universal equation of state (EOS) of the system have been theoretically studied using several model finite-ranged two-body interaction potentials 22 24]. The thermodynamic behaviour of a Fermi gas has been studied as a function of the parameters of a model finite-ranged potential of exponential type in the BCS-BEC crossover regime 23]. Two different class of finite-ranged potentials - one purely attractive such as the square well, the exponential and the Yukawa type potential, and the other having both attractive and repulsive character such as Van der Waals and dipolar potentials have been considered to explore how the depth and the spatial range of these potentials affect the pairing and molecule formation along the crossover 24]. Spin fluctuations of a strongly interacting Fermi gas across BCS-BEC crossover have been demonstrated by speckle imaging [25]. Momentum resolved photo-emission spectroscopy has been used to observe many-body pairing of a two dimensional trapped unitary Fermi gas above the transition temperature [26]. Controllable quasi-2D system across crossover has been observed with results that are beyond the mean-field level [27]. Quantized vortex ring is also observed in a unitary Fermi gas [28, 29].

The purpose of this paper is to explore the effects of the effective range of interaction on the superfluidity of a unitary Fermi gas. Towards this end, we resort to the finite-range Jost-Kohn (JK) model potential [30] which has been recently shown to account for the unitary regime. The use of this model interaction potential allows us to study the finite range effects and energy dependence of superfluid gap and density over a wide range of $a_{s}$ including the resonance limit. We use exact scattering solution of the JK potential in order to calculate momentum-dependent two-body T-matrix element for the BCS Hamiltonian. We present results on the energy-dependence of the superlfuid pairing gap over the entire range of energy for different values of $a_{s}$ and range. The gap is found to exhibit strong energy dependence in the unitary regime. We also present results on the effects of the range and $a_{s}$ on the superfluid density distribution. Our results in the zero-range limit qualitatively agree well with those for the contact interaction potential. We find that a mean gap averaged over the energy within the full width at half maximum of the gap function in the zero energy limit of our model interaction potential agrees quite well with the value calculated recently by quantum Monte Carlo simulation [14].

The paper is organised in the following way. In section 2 we discuss the standard BCS theoretical methods, introduce the Jost-Kohn model interaction potential and its exact scattering solution. In section 3 we present and discuss our results showing the effects of finite range and comparing them with those for contact interaction. We conclude and give an outlook of the work in section [4

\section{THEORETICAL METHODS}

According to the Bardeen-Cooper-Schrieffer (BCS) theory [5] of low temperature superconductivity, an attractive interaction between fermions in a quantum degenerate Fermi system leads to the formation of Cooper pairs [31] and the instability of the Fermi surface. At a critical temperature, the system then undergoes transition to superconducting phase. In solid-state electronic systems, a pair of electrons near the Fermi surface interacts by means of lattice-assisted or phonon-mediated attractive interaction, resulting in the formation of $s$-wave Cooper-pairs in spin-singlet state. The two electrons that form a Cooper-pair have equal and opposite lattice momenta. The Cooper-pair wave function is given by

$$
\psi_{0}\left(\mathbf{r}_{\mathbf{1}}, \mathbf{r}_{\mathbf{2}}\right)=\sum_{k>k_{F}} g_{\mathbf{k}} e^{i \mathbf{k} \cdot\left(\mathbf{r}_{1}-\mathbf{r}_{2}\right)}
$$

where $\mathbf{r}_{1(2)}$ refers to the position vector of the electron $1(2)$ and $g_{\mathbf{k}}$ is the wave function in momentum space. On substitution of the above relation into Schrödinger equation, one obtains

$$
\left(E-2 \epsilon_{\mathbf{k}}\right) g_{\mathbf{k}}=\sum_{k^{\prime}>k_{F}} g_{\mathbf{k}^{\prime}} V_{\mathbf{k k}^{\prime}}
$$

where $E$ is the eigen energy, $\epsilon_{k}=\hbar^{2} k^{2} / 2 \bar{m}$ ( $\bar{m}$ is reduced mass) and $V_{\mathbf{k k}^{\prime}}$ is the interaction in the momentum space. In the weakly interacting regime, $V_{\mathbf{k k}^{\prime}}$ is related to the real-space interaction potential $V_{\text {int }}(r)$ by

$$
V_{\mathbf{k k}^{\prime}}=\frac{1}{(2 \pi)^{3}} \int V_{i n t}(r) e^{-i\left(\mathbf{k}-\mathbf{k}^{\prime}\right) \cdot \mathbf{r}} d^{3} r
$$

where $\mathbf{r}=\mathbf{r}_{1}-\mathbf{r}_{2}$ is the relative position vector of the two fermions. Approximating the two-particle interaction by the Fourier transform of $V_{i n t}(r)$ into the momentum space as given above is akin to first order Born approximation 
of scattering T-matrix. In case of a conventional superconductor, $V_{\text {int }}$ is the phonon-mediated potential and is often assumed to be a space-independent contact potential. As a consequence, the momentum integration in Eq. (2) diverges. This divergence is tackled by putting a cut-off as the upper limit in the energy or wave number. In case of low temperature standard superconductors, there exists a natural cut-off which is the Debye energy or Debye wave number.

The BCS theory is applicable to superfluidity of neutral Fermi liquids or gases, albeit with some modifications that are necessary due to the absence of any natural cut-off in energy. For neutral atomic Fermi gases, $V_{\text {int }}(r)$ may be, in general, of contact or finite-range or even long-range type potential. If $V_{\text {int }}(r)$ is approximated as a contact potential represented by a delta function, $V_{\mathbf{k k}^{\prime}}$ becomes a constant that is proportional to the $s$-wave scattering length $a_{s}$. Such approximation leads to logarithmic divergence in momentum integration of gap equation. This divergence is overcome by renormalising the interaction parameter or the coupling constant by subtracting the ultraviolet divergence part from the integrand [8, 32]. However, for a short-range or finite-range interaction, such divergence problems will not arise and the gap equation or superfluid behavior will depend on the range of interactions apart from other system parameters.

Retaining only those interaction terms where two fermions interact with zero center-of-mass momentum, one can write down the reduced Hamiltonian

$$
\mathcal{H}=\sum_{k, \sigma} \epsilon_{k} \hat{n}_{k \sigma}+\sum_{k, k^{\prime}} V_{k k^{\prime}} \hat{c}_{k \uparrow}^{\dagger} \hat{c}_{-k \downarrow}^{\dagger} \hat{c}_{-k^{\prime} \downarrow} \hat{c}_{k^{\prime} \uparrow}
$$

In Hartree self consistent or generalised mean-field approach, the BCS ground state is given by generalised spin coherent state [33]

$$
\left|\psi_{G}\right\rangle=\prod_{k}\left(u_{k}+v_{k} \hat{c}_{k \uparrow}^{\dagger} \hat{c}_{-k \downarrow}^{\dagger}\right)\left|\phi_{0}\right\rangle
$$

where $\left|v_{k}\right|^{2}$ is the probability of the pair-state $(\mathbf{k},-\mathbf{k})$ being occupied and $\left|u_{k}\right|^{2}+\left|v_{k}\right|^{2}=1$. Here $\left|\phi_{0}\right\rangle$ is the vacuum state which is annihilated by $\hat{c}_{k}$. Introducing the number operator $\hat{N}=\sum_{k}\left(\hat{c}_{k \uparrow}^{\dagger} \hat{c}_{k \uparrow}+\hat{c}_{k \downarrow}^{\dagger} \hat{c}_{k \downarrow}\right)$, and a variational parameter $\theta_{k}$ such that $v_{k}=\cos \theta_{k}$ and $u_{k}=\sin \theta_{k}$, one can obtain the BCS ground state by using the variational principle [34]

$$
\delta\left\langle\psi_{G}|\mathcal{H}-\mu \hat{N}| \psi_{G}\right\rangle=0
$$

Here $\mu$ is the chemical potential which conserves the total number of particles. $\mathcal{H}$ of equation (4) can be represented in terms of pseudospin operators [33]. The value of $\langle\mathcal{H}\rangle$ is calculated by implementing equation (4) and (5). Thus we have

$$
\langle\mathcal{H}-\mu \hat{N}\rangle=\sum_{k} \xi_{k}\left(1+\cos 2 \theta_{k}\right)+\frac{1}{4} \sum_{k, k^{\prime}} V_{k k^{\prime}} \sin 2 \theta_{k} \sin 2 \theta_{k^{\prime}}
$$

where $\xi_{k}=\left(\epsilon_{k}-\mu\right)$. Taking the variation with respect to $\theta_{k}$ we have

$$
\frac{\partial}{\partial \theta_{k}}\langle\mathcal{H}-\mu N\rangle=-2 \xi_{k} \sin 2 \theta_{k}+\sum_{k^{\prime}} V_{k k^{\prime}} \cos 2 \theta_{k} \sin 2 \theta_{k^{\prime}}=0
$$

which results in

$$
\tan 2 \theta_{k}=\frac{1}{2 \xi_{k}} \sum_{l} V_{k k^{\prime}} \sin 2 \theta_{k^{\prime}}
$$

Now, defining the superfluid gap $\Delta_{k}=-\sum_{k^{\prime}} V_{k k^{\prime}} u_{k^{\prime}} v_{k^{\prime}}=-\frac{1}{2} \sum_{k^{\prime}} V_{k k^{\prime}} \sin 2 \theta_{k^{\prime}}$, the above equation can be expressed in the form of the well-known gap equation

$$
\Delta_{k}=-\frac{1}{2} \sum_{k^{\prime}} \frac{V_{k k^{\prime}} \Delta_{k^{\prime}}}{\sqrt{\Delta_{k^{\prime}}^{2}+\xi_{k^{\prime}}^{2}}}
$$

with two probability amplitudes being $u_{k}=\frac{1}{\sqrt{2}}\left(1+\xi_{k} / \sqrt{\Delta_{k}^{2}+\xi_{k}^{2}}\right)^{\frac{1}{2}}$ and $v_{k}=\frac{1}{\sqrt{2}}\left(1-\xi_{k} / \sqrt{\Delta_{k}^{2}+\xi_{k}^{2}}\right)^{\frac{1}{2}}$. 


\subsection{Finite-ranged model potential and its solution}

To study the effects of the range of interaction, we use JK interaction potential

$$
V_{i n t}(r)=-\frac{4 \hbar^{2}}{\bar{m} r_{0}^{2}} \frac{\alpha \beta^{2} e^{-2 \beta r / r_{0}}}{\left[\alpha+e^{-2 \beta r / r_{0}}\right]^{2}}
$$

where $r=\left|\mathbf{r}_{\mathbf{1}}-\mathbf{r}_{\mathbf{2}}\right|, \alpha=\sqrt{1-2 r_{0} / a_{s}}, \beta=1+\alpha, \mathbf{r}_{\mathbf{1}}$ and $\mathbf{r}_{\mathbf{2}}$ are the coordinates of two particles and the $s$-wave scattering length $a_{s}<0$.

In order to access the strongly interacting regime with a finite-ranged interaction, it is necessary to employ the exact scattering solution of the two-body problem as the first order Born approximation breaks down. In the limit of large $a_{s}$, the JK potential of eq.(11) takes the form Psöchl-Teller potential which admits an exact solution [35, 36] given by

$$
\begin{array}{r}
\psi_{k}(r)=\cosh ^{\lambda}(\kappa r) \sinh (\kappa r)_{2} F_{1}\left(\left(\frac{\lambda}{2}+\frac{i k}{2 \kappa}+\frac{1}{2}\right),\left(\frac{\lambda}{2}-\frac{i k}{2 \kappa}+\frac{1}{2}\right),\right. \\
\left.\frac{3}{2} ;-\sinh ^{2}(\kappa r)\right)
\end{array}
$$

where $\kappa=2 / r_{0}, \lambda=\frac{1}{2}(1-\sqrt{1+8 / \alpha})$ and ${ }_{2} F_{1}(a, b, c ; z)$ is the hypergeometric function. This wave function has the asymptotic form

$$
\psi_{k}(r \rightarrow \infty) \sim \pm C \cos \left(k r+\phi_{0}\right)
$$

where

$$
C=2 \Gamma(3 / 2)\left|\frac{\Gamma(-i k / \kappa) \exp (i k \log 2 / \kappa)}{\Gamma\left(\frac{\lambda+1}{2}+i \frac{k}{2 \kappa}\right) \Gamma\left(1-\frac{\lambda}{2}-i \frac{k}{2 \kappa}\right)}\right|
$$

and

$$
\phi_{0}=\arg \left[\frac{\Gamma(-i k / \kappa) \exp (i k \log 2 / \kappa)}{\Gamma\left(\frac{\lambda+1}{2}+i \frac{k}{2 \kappa}\right) \Gamma\left(1-\frac{\lambda}{2}-i \frac{k}{2 \kappa}\right)}\right]
$$

Now, we normalize the wave function $\psi_{k}^{s c t}(r) \rightarrow \psi_{k}(r) / C$ in a way so that the asymptotic form reduces to $\psi_{k}^{s c t}(r \rightarrow$ $\infty)=\sin \left(k r+\delta_{0}\right)$ where $\delta_{0}=\phi_{0}+\pi / 2$ is the $s$-wave phase shift. The effective interaction $V_{k k^{\prime}}$ in momentum space can be obtained from the exact on-the energy shell T-matrix element. Explicitly, we have

$$
V_{q}=2 \pi \int j_{0}(q r) V_{i n t}(r) \frac{\psi_{q}^{s c t}(r)}{q r} r^{2} d r
$$

where $q=\left|\mathbf{k}-\mathbf{k}^{\prime}\right|$ and $j_{0}(q r)=\sin (q r) / q r$ is the spherical Bessel function of zeroth order. To extract $V_{k k^{\prime}}$ from $V_{q}$ we write $q=\sqrt{k^{2}+k^{\prime 2}-2 k k^{\prime} \cos \theta}$. To get the exclusive dependence on $k$ and $k^{\prime}$ we must integrate $V_{q}$ over $\theta$ which then yields

$$
V_{k k^{\prime}}=\int_{\left|k-k^{\prime}\right|}^{\left(k+k^{\prime}\right)} \frac{V_{q} q}{k k^{\prime}} d q \quad k \neq 0 \neq k^{\prime}
$$

For $k=0, V_{0 k^{\prime}}=V_{q}$ and for $k^{\prime}=0, V_{k 0}=V_{q}$. Also for $k=0=k^{\prime}$ we have to take $V_{00}=V_{q}(q=0)$. From the numerical point of view we first generate a large set of discrete values of $q$ and corresponding $V_{q}$. By this procedure we obtain a two-dimensional array of $V_{k k^{\prime}}$ as a function of $k$ and $k^{\prime}$.

The JK potential is expressed in terms of two parameters, namely the $s$-wave scattering length and the effective range of a real two-body interaction potential. It is derived by inverse scattering method. So, our theoretical method is applicable to all the interacting Fermi systems that admit effective range expansion of the scaterring phase shift. So unlike various types of model potentials used in the current literature [22 24], the use of JK potential offers some advantage in numerical computation in that there is no need for the calculation of scattering length and the effective range which enter into the BCS Hamiltonian as the input parameters. But the most significant feature of the JK potential is that it can capture the resonance effect or unitary regime of interaction for which it is necessary to use exact scattering solution of the potential. However, this potential can not describe the effects of actual spatial range of a realistic potential. 

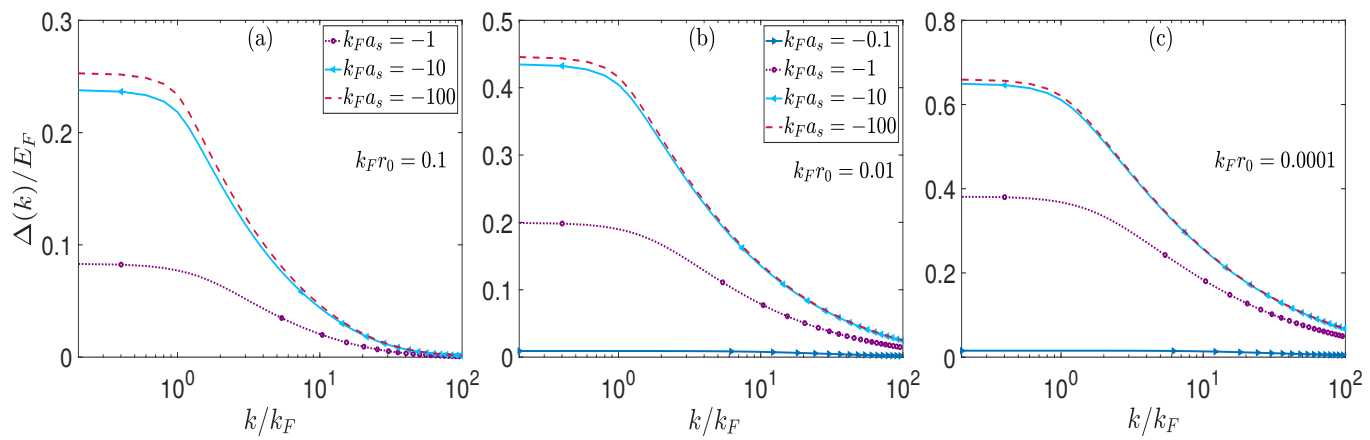

FIG. 1: The superfluid gap $\Delta(k) \equiv \Delta_{k}$ (in unit of Fermi energy $E_{F}$ ) as a function of wave number $k$ (in unit Fermi wave number) is shown for different scattering lengths $a_{s}$ and different values of the range $r_{0}$. The values of $a_{s}$ in (c) are same as in (b).
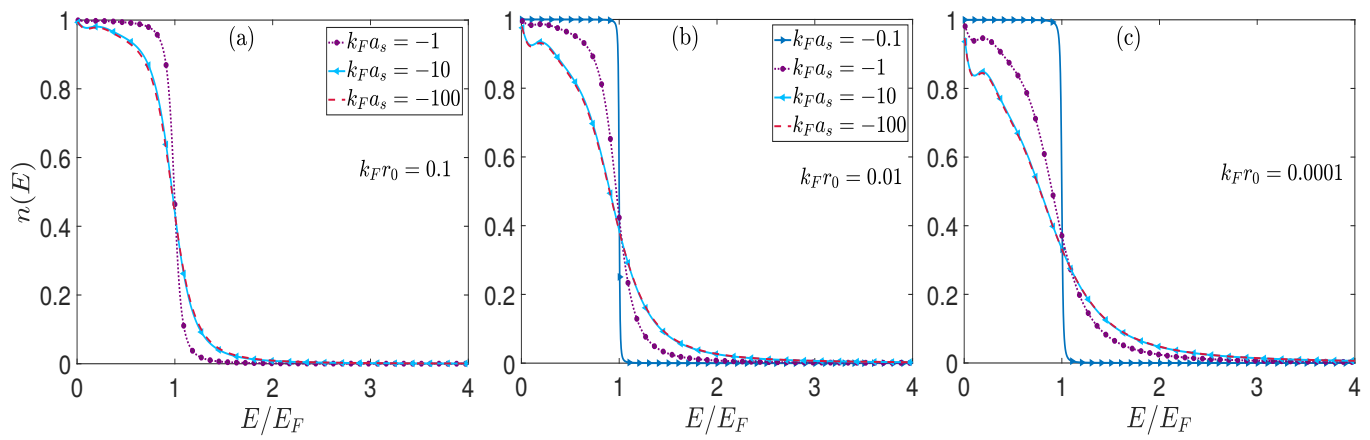

FIG. 2: The variation of superfluid density with energy $E$ (in unit of $E_{F}$ ) for the same set of parameters as in figure (1). The values of $a_{s}$ in (c) are same as in (b).

\section{2. $\quad k$-dependent gap and superfluid density}

Here we consider the superfluid gap as a function of momentum. By solving the gap equation in a self-consistent manner we calculate the $k$-dependent gap. The equation(10) is rewritten in the continuous form

$$
\Delta_{k}=-\frac{1}{2} \int \frac{V_{k k^{\prime}} \Delta_{k^{\prime}}}{\sqrt{\Delta_{k^{\prime}}^{2}+\left(\epsilon_{k^{\prime}}-\mu\right)^{2}}} \frac{d^{3} k^{\prime}}{(2 \pi)^{3}}
$$

One point worth noticing here is that we need not take into account the renormalization of the interaction term unlike that in the standard gap equation with contact potential. However, in our case the interaction in $k$-space goes to zero as $k \rightarrow \infty$. This allows us to obtain convergent solutions of $\Delta_{k}$ by a numerical iteration process.

The $k$-dependent superfluid density $n_{k}$ or equivalently $n(E)$ is given by $n(E)=\left|v_{k}\right|^{2}$ which is a dimensionless quantity with $E=\hbar^{2} k^{2} /(2 \bar{m})$. At zero temperature, the total number density $N=\frac{k_{F}^{3}}{6 \pi^{2}}$ is related to $n(E)$ by $N=\int n(E) d^{3} k /(2 \pi)^{3}$. Thus, to calculate the chemical potential $\mu$, we make use of the following equation

$$
\frac{k_{F}^{3}}{6 \pi^{2}}=\frac{1}{2} \int\left(1-\frac{\epsilon_{k}-\mu}{\sqrt{\Delta_{k}^{2}+\left(\epsilon_{k}-\mu\right)^{2}}}\right) \frac{d^{3} k}{(2 \pi)^{3}}
$$

\section{RESULTS AND DISCUSSIONS}

The wave number- or energy-dependence of superfluid gap for different values of $a_{s}$ and three values of $r_{0}$, namely, $r_{0}=0.1 k_{F}^{-1}, r_{0}=0.01 k_{F}^{-1}$ and $r_{0}=0.0001 k_{F}^{-1}$ is shown in the subplots (a), (b) and (c) of figure (1), respectively. We numerically solve the two coupled equations (17) and (18). We resort to an iterative procedure to obtain numerically the convergent values of $\Delta_{k}$ and $\mu$. We notice that $\Delta(k) \equiv \Delta_{k}$ as a function of $k$ has the peak value at $k=0$ and 

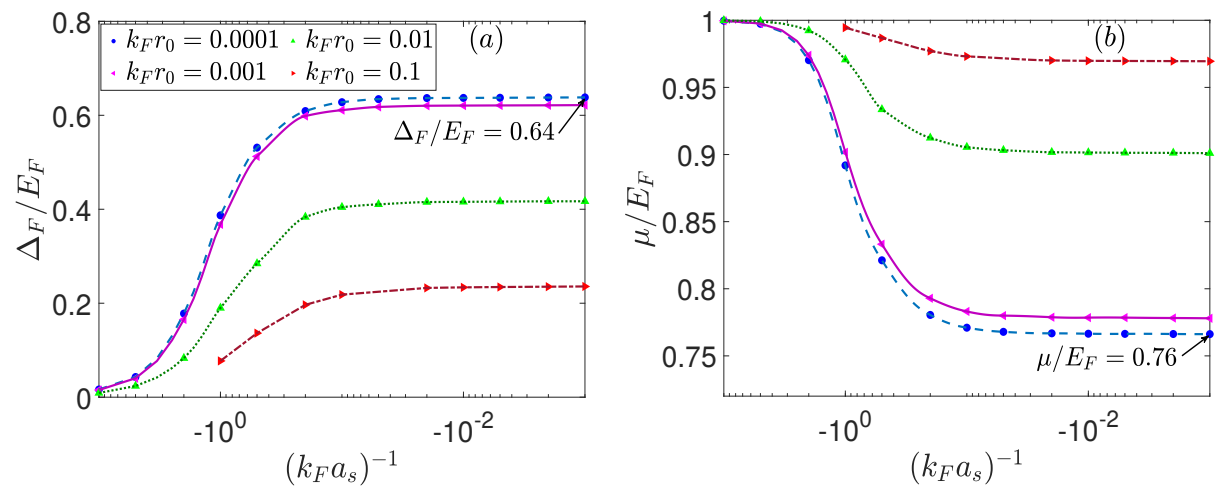

FIG. 3: Superfluid gap $\Delta_{F}$ at $k=k_{F}$ (a) and chemical potential $\mu$ (b) in Fermi energy scale are plotted as a function of inverse scattering length (in unit of $k_{F}$ ) for different ranges of interaction.

monotonically decreases as $k$ increases. Higher the value of $a_{s}$, higher is $\Delta(k)$. With decreasing $r_{0}, \Delta(k)$ increases and saturates as $r_{0} \rightarrow 0$. The figure (11) further shows that, as $r_{0}$ decreases below $0.01 \mathrm{k}_{F}^{-1}$, the results for $\Delta(k)$ in the limit $a_{s} \rightarrow-\infty$ become convergent. $\Delta(0)$ for $a_{s} \rightarrow-\infty$ is found to be close to the $k$-independent value $\Delta=0.68 E_{F}$ calculated using the zero-range pseudo-potential in the unitary limit [37].

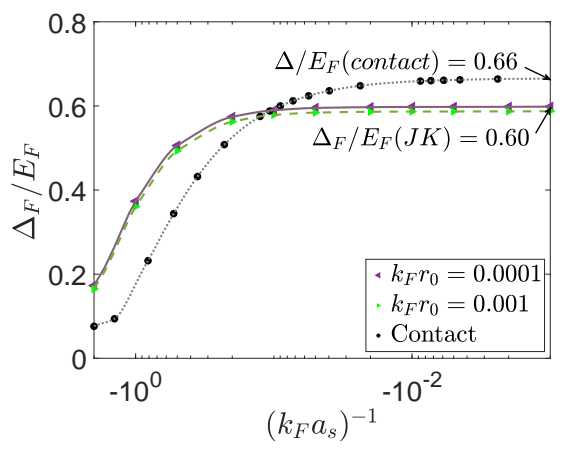

FIG. 4: Comparison between the gaps $\left(\Delta_{F}\right)$ as a function of $\left(k_{F} a_{s}\right)^{-1}$ for JK and contact potentials. The values of $\Delta_{F}$ for JK potential with $r_{0}=0.0001 k_{F}^{-1}$ and that for contact potential in the unitary limit are found to be $0.60 E_{F}$ and $0.66 E_{F}$, respectively.

The energy-dependence of superfluid density $n(E)$ for different values of $a_{s}$ and the three values of $r_{0}$ is illustrated in figure (2). For small values of $a_{s}$ in the zero-range limit, $n(E)$ resembles that of standard Fermi-Dirac distribution which then deviates as $\left|a_{s}\right|$ increases or range becomes finite.

The superfluid gap $\Delta_{F}=\Delta\left(k_{F}\right)$ at Fermi energy and chemical potential $\mu$ in unit of $E_{F}$ are plotted as a function of dimensionless interaction strength $\left(k_{F} a_{s}\right)^{-1}$ in figure (3) for different values of $r_{0}$. The variation of $\Delta_{F}$ as a function of $\left(k_{F} a_{s}\right)^{-1}$ in figure (33 ) shows qualitatively similar behavior as in the contact interaction case [37], and in the zero range limit its value is close to that of the contact case. Figure (3b) shows that, as $\left|k_{F} a_{s}\right|$ increases $\mu$ decreases. In the zero range limit of JK potential, the variation of $\mu$ with $\left(k_{F} a_{s}\right)^{-1}$ closely resembles to that for the contact interaction as given in Ref. [37]. In figure (44) we show the comparison between the variations of gap for the zero-range limit of the JK potential and the contact potential as a function $\left(k_{F} a_{s}\right)^{-1}$. Qualitatively they are similar. In the unitarity limit their quantitative values which are found to be $0.66 E_{F}$ and $0.60 E_{F}$ for contact and JK potential, respectively, agree quite well.

Figure (11) shows that the wave number dependence of $\Delta$ for finite-ranged JK potential or even in the zero range limit of this potential is quite significant. So, the question arises whether it is appropriate to compare $\Delta_{F}$ in the limit $r_{0} \rightarrow 0$ with the energy-independent gap for the contact interaction. A more reliable quantity for comparison may be a mean gap averaged over the full width at half maximum (FWHF) of the gap function. We define this energy-averaged mean gap by

$$
\tilde{\Delta}=\frac{1}{\xi} \int_{0}^{\xi} \Delta(E) d E
$$




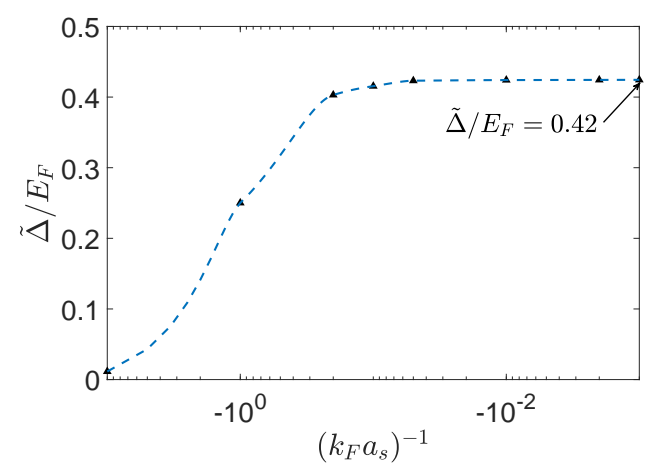

FIG. 5: $\tilde{\Delta}$ as a function of $\left(k_{F} a_{s}\right)^{-1}$ for $k_{F} r_{0}=0.0001$.

where $\xi$ is the energy corresponding to the FWHM of the $\Delta(k)$. In figure (5) we have plotted $\tilde{\Delta}$ as a function of $\left(k_{F} a_{s}\right)^{-1}$ for $r_{0}=0.0001 k_{F}^{-1}$. In the limit $a_{s} \rightarrow-\infty, \tilde{\Delta}=0.42 E_{F}$.

Most of the theoretical and experimental works on the superfluid gap of a unitary gas report the values of energyindependent gap for different system parameters. The recent experiment with superfluid ${ }^{6} \mathrm{Li}$ using momentum and specially resolved radio frequency spectroscopy [2] reports $\Delta=(0.39 \pm 0.03) E_{F}$ in the unitarity regime. There are numerous approaches to calculate the paring gap in the unitarity regime. Most of the calculations involve the quantum Monte-Carlo (QMC) technique. The first QMC calculation was done with up to 40 particles with a modified PoschlTeller interaction potential with $k_{F} r_{0} \approx 0.3\left[38\right.$ ]. The estimated gap was $\Delta \sim 0.54 E_{F}$. The inclusion of polarization correction provides a better result $\Delta \approx 0.49 E_{F}$. Diffusion Monte-Carlo calculations with improved trial function yield $\Delta=0.45 E_{F}\left[39\right.$ ] for larger number of particles with a better extrapolation to $k_{F} r_{0} \rightarrow 0$. A detailed comparison between the strong coupling theory and experimental results is made in Ref. [14]. The QMC results are in reasonable agreement with the experimental ones [40] for $r_{0}=0$. In our calculations, we numerically evaluate the gap for the entire range of energy. For the sake of comparison to other works, we choose the gap $\Delta_{F}$, that is $\Delta$ at $E=E_{F}$ and the energy-averaged gap $\tilde{\Delta}$ as defined in equation (19). We find that $\Delta_{F}=0.64 E_{F}$ in the limit $k_{F} r_{0} \rightarrow 0$ as displayed in figure $(3 \mathrm{k})$. Though this value is substantially higher than that of QMC prediction, it is slightly lower than that $\left(\Delta=0.69 E_{F}\right)$ predicted by mean-field BCS-Leggett theory [41]. However, our study shows that $\tilde{\Delta}$ as plotted in figure (5) agrees quite well with that predicted by the recent QMC calculations as well as with the experimental value. We have calculated the equation-of-states (EoS) which is given as $\bar{E}=\zeta \bar{E}_{F G}$ with $\bar{E}_{F G}$ is the ground state energy per particle of non-interacting Fermi gas and $\bar{E}$ being the energy par particle for the interacting system. We find that the Bertsch parameter $\zeta=0.69$ in the zero-range limit of the unitarity regime which has good agreement with the Monte Carlo simulation results [38].

\section{CONCLUSIONS}

In conclusion, we have demonstrated that the finite range of interaction leads to quite significant energy-dependence in the pairing gap and superfluid density both in the non-unitary and unitary regimes. The gap as a function of energy for a fixed scattering length and a fixed range is maximum at zero energy, and decreases monotonically as energy increases. For a small scattering length in the zero range limit, the energy-dependence of superfluid density resembles a step function like a standard Fermi distribution, for large scattering lengths and any value of range the density function deviates significantly from the step-like shape. The FWHM of density function is the Fermi energy. We have shown that our results on the gap and density functions approach convergent values as the range decreases below $10^{-2} k_{F}$. For the sake of comparison of our results with those for zero-range contact interaction, we have resorted in two different ways. First, we have considered the value of the gap at the Fermi energy in the zero range limit. This value is found be close to the corresponding value obtained by BCS mean-field method using contact potential. Next, we have considered the mean gap averaged over energy within FWHM of the gap in the unitary limit. This mean gap is found to be close to the results obtained by other workers using quantum Monte Carlo simulation. From a theoretical point of view, we have made use of the exact two-body scattering solution of the JK potential to obtain the energy-dependent $\mathbf{T}$-matrix element that is employed in our calculations. Unlike the case of contact interaction, the use of this exact $\mathbf{T}$-matrix element allows us to calculate accurately the energy dependence of the gap without requiring any renormalisation of the interaction. The effective range effects described in this paper may be experimentally realisable with a relatively dense cloud of ultracold fermionic atoms near a Feshbach resonance for 
which the effective range is finite, positive and likely to be tunable with an external field [30, 42]. In fact, the effective range near a narrow Feshbach resonance may become large and even negative [42 44]. In our study, we have not considered negative effective range which may also affect the pairing gap significantly [45]. However, in the known neutral $s$-wave superfluids such as neutron stars, negative effective range is unlikely to arise. Our work may serve as a precursor towards exploring quantum simulation of nuclear superfluidity using cold atoms.

Since the model potential we have used in our work, namely JK potential, is derived by inverse scattering method based on effective range expansion, the results presented here apply to all realistic interaction potentials that admit effective range expansion. There are two classes of JK potentials - one for negative scattering length which effectively describes attractive interaction and the other for positive scattering length to account for repulsive interaction. In this paper we have studied only the BCS side of the BCS-BEC crossover including the unitary regime and hence used only former type of JK potentials. While the JK potential for negative scattering length is a two-parameter potential, that corresponding to the positive scattering length is a three-parameter potential - the additional parameter being the binding energy of the last bound state of the actual potential. Therefore, in general the two potentials do not smoothly match in the unitarity limit, that is $a_{s} \rightarrow \pm \infty$. Only if one assumes that the binding energy is given by $\hbar^{2} /\left(2 \bar{m} a_{s}^{2}\right)$ ( $\bar{m}$ is the reduced mass), then the two potentials reduce to the same form in the unitarity limit 35], and so under this assumption it may be possible to explore the smooth BCS-BEC crossover with JK potentials. This also suggests that in general the BCS-BEC crossover may not be continuous or smooth when the energy of the last bound state does not go to zero in the limit $a_{s} \rightarrow \infty$. We hope to address these issues in our future communication. One of the most salient features of these potentials is that in the unitarity limit they take the form of Posch-Teller potentials which admit exact analytical solutions in one dimension. Since the partial-wave Schödinger equation is an effective one dimensional equation in one side, one can use the first odd solution of the Posch-Teller equation as the exact finite-ranged $s$-wave solution in the unitary limit within the framework of effective range expansion. So, it is expected that the use of JK potentials will enable one to study the physics in the unitarity regime more accurately.

\section{Acknowledgment}

SM is thankful to Council of Scientific and Industrial Research (CSIR), Govt. of India, for a support.

[1] I. Bloch, J. Dalibard, and W. Zwerger, "Many-body physics with ultracold gases," Rev. Mod. Phys., vol. 80, pp. 885-964, Jul 2008

[2] S. Hoinka, P. Dyke, M. G. Lingham, J. J. Kinnunen, G. M. Bruun, and C. J. Vale, "Resonantly interacting fermions in a box," Nat. Phys., vol. 13, pp. 943-946, 2017.

[3] C. C. N. Kuhn, S. Hoinka, I. Herrera, P. Dyke, J. J. Kinnunen, G. M. Bruun, and C. J. Vale, "High-frequency sound in a unitary fermi gas," Phys. Rev. Lett., vol. 124, p. 150401, Apr 2020.

[4] J. Dalibard, F. Gerbier, G. Juzeliūnas, and P. Öhberg, "Colloquium: Artificial gauge potentials for neutral atoms," Rev. Mod. Phys., vol. 83, pp. 1523-1543, Nov 2011.

[5] J. Bardeen, L. N. Cooper, and J. R. Schrieffer, "Theory of superconductivity," Phys. Rev., vol. 108, pp. 1175-1204, Dec 1957.

[6] C. Chin, R. Grimm, P. Julienne, and E. Tiesinga, "Feshbach resonances in ultracold gases," Rev. Mod. Phys., vol. 82, pp. 1225-1286, Apr 2010.

[7] S. J. J. M. F. Kokkelmans, J. N. Milstein, M. L. Chiofalo, R. Walser, and M. J. Holland, "Resonance superfluidity: Renormalization of resonance scattering theory," Phys. Rev. A, vol. 65, p. 053617, May 2002.

[8] M. Randeria, J.-M. Duan, and L.-Y. Shieh, "Bound states, cooper pairing, and bose condensation in two dimensions," Phys. Rev. Lett., vol. 62, pp. 981-984, Feb 1989.

[9] R. Micnas, J. Ranninger, and S. Robaszkiewicz, "Superconductivity in narrow-band systems with local nonretarded attractive interactions," Rev. Mod. Phys., vol. 62, pp. 113-171, Jan 1990.

[10] M. Randeria, N. Trivedi, A. Moreo, and R. T. Scalettar, "Pairing and spin gap in the normal state of short coherence length superconductors," Phys. Rev. Lett., vol. 69, pp. 2001-2004, Sep 1992.

[11] M. Drechsler and W. Zwerger, "Crossover from bcs-superconductivity to bose-condensation," Annalen der Physik, vol. 504, no. 1 , pp. $15-23$.

[12] M. Casas, J. M. Getino, M. de Llano, A. Puente, R. M. Quick, H. Rubio, and D. M. van der Walt, "Bcs-bose model of exotic superconductors: Generalized coherence length," Phys. Rev. B, vol. 50, pp. 15945-15952, Dec 1994.

[13] M. Horikoshi, M. Koashi, H. Tajima, Y. Ohashi, and M. Kuwata-Gonokami, "Ground-state thermodynamic quantities of homogeneous spin-1/2 fermions from the bcs region to the unitarity limit," Phys. Rev. X, vol. 7, p. 041004 , Oct 2017.

[14] P. van Wyk, H. Tajima, D. Inotani, A. Ohnishi, and Y. Ohashi, "Superfluid fermi atomic gas as a quantum simulator for the study of the neutron-star equation of state in the low-density region," Phys. Rev. A, vol. 97, p. 013601, Jan 2018. 
[15] M. Horikoshi and M. Kuwata-Gonokami, "Cold atom quantum simulator for dilute neutron matter," Int. J. Mod. Phys. E, vol. 28, no. 01n02, p. 1930001, 2019.

[16] X. Y. Yin, H. Hu, and X.-J. Liu, "Cluster formation in two-component fermi gases," Phys. Rev. Lett., vol. 123, p. 073401, Aug 2019 .

[17] G. A. Baker, "Neutron matter model," Phys. Rev. C, vol. 60, p. 054311, Oct 1999.

[18] S. Heckel, P. P. Schneider, and A. Sedrakian, "Light nuclei in supernova envelopes: A quasiparticle gas model," Phys. Rev. $C$, vol. 80, p. 015805, Jul 2009.

[19] G. Baym, T. Hatsuda, T. Kojo, P. D. Powell, Y. Song, and T. Takatsuka, "From hadrons to quarks in neutron stars: a review," Rep. Prog. Phys., vol. 81, p. 056902, Mar 2018.

[20] N. Navon, S. Nascimbène, F. Chevy, and C. Salomon, "The equation of state of a low-temperature fermi gas with tunable interactions," vol. 328, no. 5979, pp. 729-732, 2010.

[21] M. M. Forbes, S. Gandolfi, and A. Gezerlis, "Resonantly interacting fermions in a box," Phys. Rev. Lett., vol. 106, p. 235303, Jun 2011.

[22] M. M. Forbes, S. Gandolfi, and A. Gezerlis, "Effective-range dependence of resonantly interacting fermions," Phys. Rev. $A$, vol. 86, p. 053603, Nov 2012.

[23] S. F. Caballero-Benítez, P. R., and V. Romero-Rochín, "The contact in the bcs-bec crossover for finite range interacting ultracold fermi gases," Phys. Lett. A, vol. 377, pp. 1756-1759, 2013.

[24] S. F. Neri, E. andb Caballero-Benítez, V. Romero-Rochín, and P. R., "The contact in the bcs-bec crossover for finite range interacting ultracold fermi gases," Phys. Scr., vol. 95, p. 034013, 2020.

[25] C. Sanner, E. J. Su, A. Keshet, W. Huang, J. Gillen, R. Gommers, and W. Ketterle, "Speckle imaging of spin fluctuations in a strongly interacting fermi gas," Phys. Rev. Lett., vol. 106, p. 010402, Jan 2011.

[26] M. Feld, B. Frohlich, E. Vogt, M. Koschorreck, and M. Khol, "Observation of a pairing pseudogap in a two-dimensional fermi gas," Nature, vol. 480, pp. 75-78, Dec 2011.

[27] V. Makhalov, K. Martiyanov, and A. Tquasi-2d fermi and bose gases," Phys. Rev. Lett., vol. 112, p. 045301, Jan 2014.

[28] T. Yefsah, A. T. Sommer, L. W. Ku, Mark J. H.and Cheuk, W. Ji, W. S. Bakr, and M. W. Zwierlein, "Heavy solitons in a fermionic superfluid," Nature, vol. 499, pp. 426-430, Jul 2013.

[29] A. Bulgac, M. M. Forbes, M. M. Kelley, K. J. Roche, and G. Wlazłowski, "Quantized superfluid vortex rings in the unitary fermi gas," Phys. Rev. Lett., vol. 112, p. 025301, Jan 2014.

[30] S. Mal, K. Adhikary, D. Sardar, A. K. Saha, and B. Deb, "Modeling atom-atom interactions at low energy by jost-kohn potentials," J. Phys. B: At. Mol. Opt. Phys., vol. 52, p. 165004, Jul 2019.

[31] L. N. Cooper, "Bound electron pairs in a degenerate fermi gas," Phys. Rev., vol. 104, pp. 1189-1190, Nov 1956.

[32] B. Deb, A. Mishra, H. Mishra, and P. K. Panigrahi, "Interior gap superfluidity in a two-component fermi gas of atoms," Phys. Rev. A, vol. 70, p. 011604, Jul 2004.

[33] A. Montorsi and V. Penna, "Dynamics of the hubbard model: A general approach by the time-dependent variational principle," Phys. Rev. B, vol. 55, pp. 8226-8239, Apr 1997.

[34] P.-G. de Gennes, Superconductivity of Metals and Alloys. Advanced book classics.

[35] B. Deb, "Finite-range model potentials for resonant interactions," Int. J. Mod. Phys. B, vol. 30, no. 08, p. 1650036, 2016.

[36] S. Flügge, Practical Quantum Mechanics. Classics in Mathematics.

[37] B. Deb, "Light scattering in cooper-paired fermi atoms," J Phys. B: At., Mol. Opt. Phys., vol. 39, pp. 529-551, Dec 2005.

[38] J. Carlson, S.-Y. Chang, V. R. Pandharipande, and K. E. Schmidt, "Superfluid fermi gases with large scattering length," Phys. Rev. Lett., vol. 91, p. 050401, Jul 2003.

[39] J. Carlson and S. Reddy, "Superfluid pairing gap in strong coupling," Phys. Rev. Lett., vol. 100, p. 150403, Apr 2008.

[40] S. Hoinka, P. Dyke, M. G. Lingham, J. J. Kinnunen, G. M. Bruun, and C. J. Vale, "Goldstone mode and pair-breaking excitations in atomic fermi superfluids," Nat. Phys., vol. 13, pp. 943-946, 2017.

[41] A. J. Leggett, "Diatomic molecules and cooper pairs," in Modern Trends in the Theory of Condensed Matter (A. Pekalski and J. A. Przystawa, eds.), (Berlin, Heidelberg), pp. 13-27, Springer Berlin Heidelberg, 1980.

[42] P. Dyke, S. E. Pollack, and R. G. Hulet, "Finite-range corrections near a feshbach resonance and their role in the efimov effect," Phys. Rev. A, vol. 88, p. 023625, Aug 2013.

[43] C. L. Blackley, P. S. Julienne, and J. M. Hutson, "Effective-range approximations for resonant scattering of cold atoms," Phys. Rev. A, vol. 89, p. 042701, Apr 2014.

[44] E. L. Hazlett, Y. Zhang, R. W. Stites, and K. M. O'Hara, "Realization of a resonant fermi gas with a large effective range," Phys. Rev. Lett., vol. 108, p. 045304, Jan 2012.

[45] J. Hu, F. Wu, L. He, X.-J. Liu, and H. Hu, "Theory of strongly paired fermions with arbitrary short-range interactions," Phys. Rev. A, vol. 101, p. 013615, Jan 2020. 